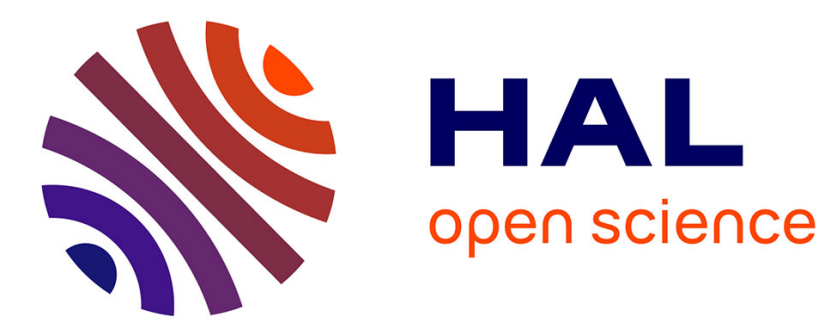

\title{
Restricted Conformation Dynamics of Single Functionalized Perylene Bisimide Molecules on SiO2 Surfaces and in Thin Polymer Films
}

Danny Kowerko, Jörg Schuster, Christian von Borczyskowski

\section{- To cite this version:}

Danny Kowerko, Jörg Schuster, Christian von Borczyskowski. Restricted Conformation Dynamics of Single Functionalized Perylene Bisimide Molecules on SiO2 Surfaces and in Thin Polymer Films. Molecular Physics, 2009, 107 (18), pp.1911-1921. 10.1080/00268970902758631 . hal-00514964

\author{
HAL Id: hal-00514964 \\ https://hal.science/hal-00514964
}

Submitted on 4 Sep 2010

HAL is a multi-disciplinary open access archive for the deposit and dissemination of scientific research documents, whether they are published or not. The documents may come from teaching and research institutions in France or abroad, or from public or private research centers.
L'archive ouverte pluridisciplinaire HAL, est destinée au dépôt et à la diffusion de documents scientifiques de niveau recherche, publiés ou non, émanant des établissements d'enseignement et de recherche français ou étrangers, des laboratoires publics ou privés. 


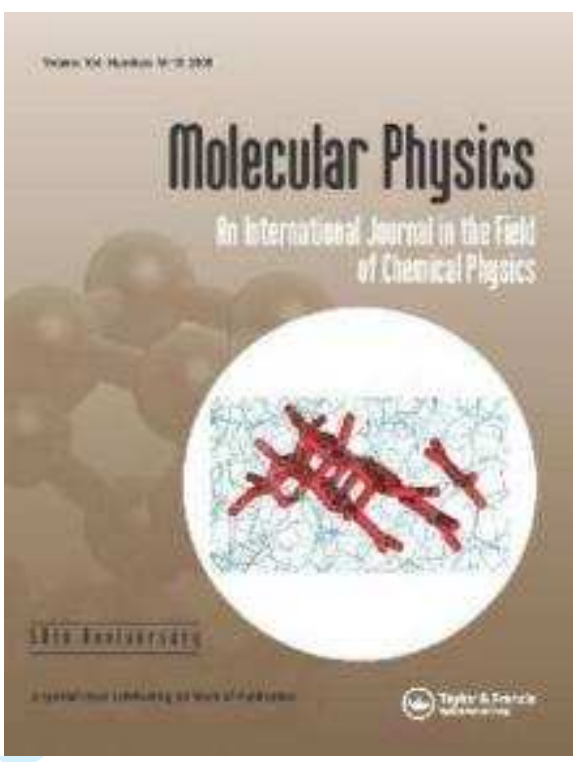

\section{Restricted Conformation Dynamics of Single Functionalized Perylene Bisimide Molecules on $\mathrm{SiO}_{2}$ Surfaces and in Thin Polymer Films}

\begin{tabular}{|c|c|}
\hline Journal: & Molecular Physics \\
\hline Manuscript ID: & TMPH-2008-0378.R1 \\
\hline Manuscript Type: & Special Issue Paper - Spectral Dynamics \& Related Topics \\
\hline $\begin{array}{r}\text { Date Submitted by the } \\
\text { Author: }\end{array}$ & 13-Jan-2009 \\
\hline Complete List of Authors: & $\begin{array}{l}\text { Kowerko, Danny; TU Chemnitz, institute of physics } \\
\text { Schuster, Jörg; TU Chemnitz, institute of physics } \\
\text { von Borczyskowski, Christian; TU Chemnitz, institute of physics }\end{array}$ \\
\hline Keywords: & $\begin{array}{l}\text { single molecule spectroscopy, conformation dynamics, perylene } \\
\text { bisimides, polymer thin film, surface adsorption }\end{array}$ \\
\hline
\end{tabular}

\section{s) ScholarONE" \\ Manuscript Central}




\title{
Polymer Films
}

\author{
Danny Kowerko ${ }^{1 *}$, Jörg Schuster ${ }^{1}$ \\ and Christian von Borczyskowski ${ }^{1}$
}

1) Institute of Physics and nanoMA (Center for nanostructured Materials and Analytics), Chemnitz University of Technology, 09107 Chemnitz, Germany

*) Corresponding author, Institute of Physics and nanoMA, Chemnitz University of Technology, 09107 Chemnitz, Germany, danny.kowerko@physik.tu-chemnitz.de 


\begin{abstract}
Intramolecular conformational dynamics caused by the bay groups of perylene bisimide (PBI) molecules have been investigated by single molecule spectroscopy in thin PMMA polymer films and on $\mathrm{SiO}_{2}$ surfaces. All dynamic processes occur in a jump-like fashion on $\mathrm{SiO}_{2}$ but in a likewise continuous way in PMMA. Surface attachment on $\mathrm{SiO}_{2}$ is accomplished by pyridyl functionalisation of PBI demonstrating a nearly perpendicular orientation of the long axis of perylene as has been concluded from polarisation experiments. This orientation is subject to fluctuations of the molecular orientation and/or conformation, which are considerably reduced in PMMA. According to static and time dependent fluorescence spectra at least 2 different conformations have been identified.
\end{abstract}




\section{Introduction}

Single molecule detection has been extensively used to investigate both intramolecular interactions such as interconversion between different conformations [1,2,3] or subtle intermolecular interactions thus probing static and dynamic matrix properties e. g. of glasses [4,5] liquid layers [6,7] and films [8]. Perylene bisimide (PBI) type molecules are one of those dye molecules which show on one hand a sufficiently high photostability. On the other hand they are due to various (bay) groups subject to intramolecular dynamics $[9,10]$. In a recent publication [11] a comparison of time resolved fluorescence and molecular dynamics calculations has demonstrated, that these bay groups control the formation and interconversion of two limiting classes of conformations. Additionally, different conformations might give rise to twisting of the perylene core itself $[12,13]$. It is evident, that the realisation of conformations and the related dynamics will be controlled by external restrictions such as embedding into various polymers $[14,15]$. This way PBI might also be used as a type of microscopic probe for matrix properties and dynamics as has been already demonstrated successfully [16,17].

PBI type molecules can be functionalised e. g. via substituted pyridyl rings [18]. Making use of different types of (multi-)pyridyl substituted PBI a whole class of supramolecular architectures has been realized via coordination chemistry and characterized also on a single molecule level [19]. The ability to form complexes via the pyridyl groups of differently pyridyl-substituted PBI molecules has been recently extended to the formation of complexes between PBI dyes and colloidal CdSe/ZnS semiconductor quantum dots (QDs) via selfassembly [20]. In that paper we successfully demonstrate, how, with respect to the QD surface, relative orientations of complexed pyridyl- or terpyridyl-functionalized PBI molecules can be derived from Förster resonance energy transfer (FRET) processes. Besides FRET we also discussed in that paper the role of PBI induced CdSe/ZnS luminescence quenching caused by NON-FRET transfer processes.

In the present paper we like to extend the concept of controlled attachment of single PBI dyes to surfaces such as $\mathrm{SiO}_{2}$. Besides the characterisation of the surface attachment we will investigate the remaining mobility of $\mathrm{PBI}$ and the influence of the attachment on the (intramolecular) conformation dynamics. We will compare the results for "quasi" free molecules on $\mathrm{SiO}_{2}$ with those in a strictly hindering PMMA polymer thin film. Results on a single molecule level will be obtained by simultaneous detection of time dependent fluorescence (spectral diffusion), polarisation (rotational motion) and fluorescence intensity fluctuations. 


\section{Experimental}

The synthesis of pyridyl-substituted perylene bisimide (PP) and dialkyl-substituted perylene bisimide (DAP) investigated in the present experiments is described elsewhere [18,19]. PP and DAP molecules are shown in Figure 1. DAP serves merely as a reference system to provide in comparison with PP information on the degree of pyridyl mediated surface attachment of PP. Substituting four phenoxy groups at the carbocyclic scaffold further on called "bay groups" $[18,19]$ (one of these four groups is marked in Figure 1, left) enhances solvation in toluene (Merck, spectroscopic grade). For ensemble experiments $10^{-7} \mathrm{M}$ stock solutions of PBI dye molecules were prepared in toluene. Hellma QS suprasil quartz cuvettes and caps were cleaned thoroughly with the special HF containing detergent "Hellmanex II" and Piranha solution $\left(1: 1 \mathrm{H}_{2} \mathrm{SO}_{4}: \mathrm{H}_{2} \mathrm{O}_{2}\right)$ in a supersonic bath at $70^{\circ} \mathrm{C}$ for several minutes and 2 $\mathrm{h}$ for the caps, respectively. Exact concentrations were determined with a Varian "Cary Scan 100" UV/Vis spectrometer, while luminescence spectroscopy was performed with a Varian "Cary Eclipse" fluorescence spectrometer.

Figure 1 Molecular structures of pyridyl- and alkyl-functionalized PBI derivatives PP (left) and DAP (right). One of the four phenoxy groups in the PBI's so-called bay area is exemplarily marked at the PP molecule.

For single molecule experiments samples, pipettes and vials were thoroughly cleaned by supersonic treatment in piranha solution $\left(1: 1 \mathrm{H}_{2} \mathrm{O}_{2}-\mathrm{H}_{2} \mathrm{SO}_{4}\right.$ solution) followed by careful rinsing with Millipore DI water. The purity of the solvent and pipettes has been assured via spincoating toluene and subsequent imaging of the sample with a homebuilt fluorescence wide field microscope, Accordingly single particle sample preparation has been achieved by spin coating a $5 \cdot 10^{-11} \mathrm{M}$ or even lower concentrated solution of dye molecules onto a $\mathrm{Si} / \mathrm{SiO}_{2}$ substrate (100 $\mathrm{nm} \mathrm{SiO}_{2}$ thickness).

Principally the same procedure was used for sample preparation of PP embedded in polymethylmethacrylat (PMMA, Aldrich). An amount of $20 \mathrm{mg}$ PMMA was diluted in $3 \mathrm{ml}$ of toluene and spincoated onto the $\mathrm{Si} / \mathrm{SiO}_{2}$ substrate without dye molecules to controle the purity of PMMA, In the next step some microliters of a PP stock solution $\left(\sim 2 \cdot 10^{-7} \mathrm{M}\right.$, dissolved in toluene) were added to the toluene/PMMA solution to obtain a $10^{-10} \mathrm{M}$ concentration of PP. The latter is spincoated to the substrate in the same way as for single molecule samples on $\mathrm{Si} / \mathrm{SiO}_{2}$ without PMMA.
Deleted: Both perylene bisimide (PBI) molecules have been dissolved in toluene (Merck, spectroscopic grade).

Deleted: $\mathrm{p}$

Deleted: Incorporation of

Deleted: solubilising

Deleted: substituents

Deleted:

Deleted: in the so-called bay area

Deleted: exemplarily

Deleted: a

Deleted: allows perylene bisimide (PBI) molecules to be dissolved

Deleted: the

Deleted: a

Deleted: By means of

Deleted: the pure

Deleted: solvent

Deleted: the purity of the solvent and pipettes has been assured

Deleted: about

Deleted: ver

Deleted: verify the cleanness

Deleted: poured

Deleted: vial

Deleted: gain 
Single molecule experiments have been performed in a home built laser scanning confocal microscope at room temperature. The sample was excited by a cw-NdYAG laser (Spectra Physics) using an excitation wavelength of $532 \mathrm{~nm}$. The laser beam was focused by a 100x objective lens (Zeiss "EC Epiplan Neofluar") with a numerical aperture of NA $=0.9$. Assuming a diffraction limited focal spot, the excitation power density was adjusted to 0.6 $\mathrm{kW} / \mathrm{cm}^{2}$. An avalanche photo diode (Perkin Elmer, "SPCM-AQR-16") with a very low dark count rate served as photo detector. Laser excitation light was blocked by an Omega optical long pass filter "542 ALP" in the detection path. In front of the detector a 3:1 beamsplitter couples part of the light into a spectrometer (Acton Research Corp “"Acton SpectraPro-275") equipped with a liquid nitrogen cooled CCD camera (Princeton Instuments),

For polarisation resolved experiments a homebuilt wide field microscope with a Wollaston prism inserted into the fluorescence detection path in front of the $\mathrm{CCD}_{\mathrm{s}}$ Excitation light supplied by the $515 \mathrm{~nm}$ laser line of an argon ion laser (Spectra Physics), was stabilized by an electro-optical modulator (Conoptics). Circular polarized light was achieved by inserting a $\lambda / 4$ plate (Thorlabs, "AQWP05M-630") into the excitation beam followed by a laser line filter at $515 \mathrm{~nm}$ (Omega). Laser light was directed to an objective lens (the same as in the confocal microscope) via a dichroic mirror (Omega "525 DRLP"). Fluorescence, was focused onto an intensified frame transfer camera (Pentamax Roper Scientific), whereas excitation laser light was suppressed by a long pass filter (Omega "3RD530LP”).

Deleted: $\mathrm{I}$
Deleted: he illumination of $\mathrm{t}$
Deleted: carried
Deleted: out
Deleted: $\mathrm{cw}-$

Deleted: luminescence

Deleted: ,

Deleted:

Deleted: as detector

\begin{tabular}{|c|}
\hline Deleted: in \\
\hline Deleted: \\
\hline Deleted: a \\
\hline Deleted: camera was used \\
\hline Deleted: , \\
\hline Deleted: , \\
\hline Deleted: Formation of c \\
\hline Deleted: path \\
\hline Deleted: is \\
\hline Deleted: , \\
\hline Deleted: $\mathrm{t}$ light \\
\hline $\begin{array}{l}\text { Deleted: DAP serves as reference } \\
\text { system since the surface orientation of } \\
\text { this type of molecules is expected to be } \\
\text { rather arbitrary due to its lack of the } \\
\text { functional pyridyl group. For discussion } \\
\text { of conformational dynamics, most } \\
\text { experimental results presented here have } \\
\text { been performed on PP molecules, } \\
\text { whereat pyridyl specific surface } \\
\text { adsorption reduces the occurrence of } \\
\text { molecular rotation.II }\end{array}$ \\
\hline
\end{tabular}




\section{Results}

Ensemble fluorescence spectra of PP are shown in Figure 2 in toluene together with the sumspectra of all single PP molecule spectra in PMMA and on $\mathrm{SiO}_{2}$, respectively. The fluorescence maxima are at $605 \mathrm{~nm}$ (toluene) and $594 \mathrm{~nm}$ (PMMA). The sum-spectrum for PP on $\mathrm{SiO}_{2}$ shows a strong maximum at $605 \mathrm{~nm}$ (with a weak shoulder at $\approx 595 \mathrm{~nm}$ ) and is shifted with respect to PMMA by $11 \mathrm{~nm}$ to the red. The range of fluorescence maxima is comparable to results observed for very similar PBI molecules in chloroform (608 $\mathrm{nm})$ [16], in a 60 : 40 mixture of $\mathrm{CHCl}_{3}-\mathrm{CH}_{3} \mathrm{OH}(620 \mathrm{~nm})$ [21], in $\mathrm{CH}_{2} \mathrm{Cl}_{2}(618 \mathrm{~nm})$ [22], and toluene $(606 \mathrm{~nm})$ [9]. The spectrum in toluene shows a pronounced vibronic side band (as has been observed in all other solution spectra), which is less pronounced for PP in PMMA and on $\mathrm{SiO}_{2}$.

Figure 2 Fluorescence spectra of PP in different environments. Black (PMMA) and red $\left(\mathrm{SiO}_{2}\right)$ spectra result from summing up the respective single molecule spectra recorded during a 1 min measurement period. Excitation wavelength was $53 \overline{\mathbf{n m}}$.

Single molecule experiments reveal that upon spincoating a toluene or toluene/PMMA solution with the same initial concentration of PP, the final concentration of emitting single molecules is by about a factor of 2.5 larger in case of $\mathrm{SiO}_{2}$ as compared to PMMA. This ratio may be even larger, since the average fluorescence intensity of single $\mathrm{PP}$ on $\mathrm{SiO}_{2}$ is under the same experimental conditions by a factor of two smaller than that of PP in PMMA.

Inspecting the spectra of single PP molecules with bin times of $1 \mathrm{~s}$ reveals that spectral positions, spectral shapes and fluorescence intensities are time-dependent. This is shown in Figure 3 for two representative examples in case of $\mathrm{PP}$ on $\mathrm{SiO}_{2}$ and in $\mathrm{PMMA}$, respectively.

Deleted: time

Deleted: typical

Figure 3 Temporal evolution of PP fluorescence on $\mathrm{SiO}_{2}$ (left) and in PMMA (right). The integrated fluorescence intensity (determined by a fit to two Gaussian lines at the fluorescence origin and the vibronic side band, respectively) are shown as traces on the right of each spectral scan. The centres of the two fitted spectral positions (traces on the left) of the respective spectra are also indicated. After about 55

| s (left) and $65 \mathrm{~s}$ (right) PP molecules are photobleached permanently

The spectral distribution of the fluorescence maxima $\lambda_{1}$ (of all molecules) is shown in Figure 4. The related non-Gaussian distribution is broader than $25 \mathrm{~nm}$ (PMMA) and $30 \mathrm{~nm}\left(\mathrm{SiO}_{2}\right)$ showing at least 2 distinct maxima at about 585 and $600 \mathrm{~nm}$ (PMMA) and 592 and $605 \mathrm{~nm}$ $\left(\mathrm{SiO}_{2}\right)$, respectively. These values are - as expected - in agreement with the maxima obtained 
Figure 4 Distribution of fluorescence maxima $\lambda_{1}$ position for single $\mathrm{PP}$ molecules on $\mathrm{SiO}_{2}$ and in thin PMMA films. The insets show the spectral position of $\lambda_{2}$ vibrational bands relative to $\lambda_{1}$. Broken lines indicate possible PP conformations below and above $592 \mathrm{~nm}$, which corresponds to the wavelength with the highest fluorescence intensity (see Figure 7).

Though a precise determination of the energetic position of the vibrational band is due to the limited signal to noise ratio difficult, we qualitatively observe that $\Delta \lambda=\lambda_{2}-\lambda_{1}$ as defined by the wavelength difference between the main and side band (fit to two Gaussian bands) is close to $40 \mathrm{~nm}\left(\mathrm{SiO}_{2}\right)$ and $35 \mathrm{~nm}$ (PMMA), respectively.

As is clearly demonstrated in Figure 3, spectral positions and fluorescence intensities fluctuate on time scales of seconds. The distribution of spectral jump widths is shown in Figure 5. For both matrices the width of the distribution is close to $5 \mathrm{~nm}$ (at half height), but is slightly asymmetric with an extension of large jumps towards long wavelengths (PMMA) and short wavelengths $\left(\mathrm{SiO}_{2}\right)$. This is in qualitative agreement (see Figure 4) with an increased probability to find PP molecules at long wavelengths for $\mathrm{SiO}_{2}$ as compared to PMMA. A larger spectral diffusion range covered during the observation time (see insets in Figure 5) is found for $\mathrm{SiO}_{2}$ as compared to PMMA. From the representative molecules (see Figure 3) it is additionally evident that spectral shifts or fluorescence intensities of PP in PMMA develop more gradually, while they occur more sudden in case of $\mathrm{SiO}_{2}$.

| Figure 5 Distribution (on two probability scales) of spectral jumps occurring between two subsequently recorded spectra (1s binning time for each spectrum). The insets show the largest spectral range $\Delta \lambda_{\max }$ covered by one individual molecule during the total observation time.

One of the principle differences between $\mathrm{SiO}_{2}$ and PMMA is, that PMMA is a polymer at room temperature, which will hinder translational or rotational motion of the embedded molecules. This condition will be relaxed in case of $\mathrm{SiO}_{2}$. For both environments we did on a $100 \mathrm{~s}$ time scale not detect any translational motion larger than $20 \mathrm{~nm}$, which is the accuracy to determine lateral positions via confocal techniques [23]. Since PP has a strong optical transition dipole moment parallel to the long molecular axis [24], time dependent polarisation measurements provide information about rotational motion. The respective fluorescence intensities in the two orthogonal in-plane polarisation channels 1 and 2 are shown in Figure 6 for several single molecules. The related angle $\phi$ of the in-plane orientation of the projected transition dipole orientation (see scheme at Figure 8) is also shown. A direct comparison

Deleted: of

\begin{tabular}{|l|}
\hline Deleted: \\
\hline Deleted: (on two probability scales) \\
\hline Deleted: observed \\
\hline Deleted: for \\
\hline Deleted: \\
\hline Deleted: forms \\
\hline Deleted: solid glass \\
\hline Deleted: on a 100 s time scale \\
\hline
\end{tabular}


between results for PMMA and $\mathrm{SiO}_{2}$ reveals, that we can only in case of $\mathrm{SiO}_{2}$ observe - if at all - sudden jumps in polarisation whereas we observe for PMMA only gradual changes in polarisation

Figure 6 Representative time resolved polarisation intensities for 3 single molecules of $\mathrm{PP}$ on $\mathrm{SiO}_{2}$ surfaces ((a) - (c)) and in a PMMA thin film ((d) - (f)), respectively. Channel 1 and 2 correspond to fluorescence intensities of the two perpendicular in-plane polarisation directions (top trace). $\phi$ (middle trace) corresponds to the time dependence of the projection angle $\phi$ (see Figure 8). The lowest trace shows the probability distribution of the related $\phi$-time trace above).
Deleted: for PMMA

Deleted:

\begin{tabular}{|l|}
\hline Deleted: Typical \\
\hline Deleted: \\
\hline Deleted: resolved in time \\
Deleted: s \\
\hline Deleted: graph \\
Deleted: (middle trace
\end{tabular}

\section{Discussion}

Recent publications related to PBI type molecules have concentrated on conformational dynamics in solution via ensemble $[10,11,25,26]$, single molecule experiments in polymers $[9,27]$ and in Shpol'skii matrices [19]. One of the related questions is the distortion from planarity of the perylene chromophoric unit and the influence of the bay groups on the formation of different conformations. Due to steric hindrance bay groups will control the degree of distortion of these molecules [9,26]. In recent studies $[10,11]$ the influence of the phenoxy bay groups on the formation of PBI conformations has been investigated extensively. It has been observed that the orientation of the bay groups with respect to the perylene bisimide unit determines the type of conformation. In this study Fron and coworkers [10,11] 
elucidated the photophysical properties of these perylene bisimides in great detail by single photon timing and femtosecond transient absorption experiments in close comparison with molecular dynamics (MD) calculations. To some extent we will relate our results to their, findings by comparing them on a single molecule level with our results in two different matrices.

As has been shown extensively, the different conformations of PP will influence electronic energies, fluorescence quantum yields and spectral properties such as the vibronic structure. While ensemble experiments always give the superposition of all the realised conformations, single molecule experiments are well suited to identify individual conformations and, even more important, allow to investigate directly possible transitions among such conformations [9]. Additionally, it has been extensively shown in recent and present experiments that spectral positions, optical polarisations and fluorescence decay times fluctuate on time scales of ms and longer. Observed fluctuations might be - besides conformational changes - also the result of spatially inhomogeneous and time dependent dye-polymer interactions [5]. Such properties have been exploited to investigate intensively dynamics in glasses $[4,15]$. With respect to PBI type dyes it has e. g. been found that the spatial inhomogeneity is larger for Zeonex than for PMMA matrices [16].

As already mentioned in the introduction another feature of PBI type dyes is of interest, namely to form supramolecular assemblies and polymers via pyridyl functionalisation [28]. PP is one of the simplest forms of such a functionalized PBI. Discussing the present results for single PP molecules we like to concentrate on the aspects, how the pyridyl functionalisation influences as a function of the environment the types of molecular conformations and the related interconversion dynamics. We have chosen PMMA thin films and a planar $\mathrm{SiO}_{2}$ surface, which are different in that aspect that $\mathrm{SiO}_{2}$ should allow for a more free evolution of the molecular conformations and orientations (gas phase type environment), while PMMA (as a prototype of an amorphous solid) is expected to hinder strongly molecular dynamics in general.

From the comparison of the fluorescence intensity distribution of PP molecules on $\mathrm{SiO}_{2}$ and in PMMA two observations immediately emerge, namely (i) when starting with the same concentration before spincoating the final number of $\mathrm{PP}$ molecules on $\mathrm{SiO}_{2}$ is by a factor of 2.5 larger than in PMMA but (ii) the average PL intensity of PP on $\mathrm{SiO}_{2}$ is by a factor 2
Deleted: se reported

Deleted: It became evident, that

Deleted:

Deleted: determine 
smaller than in the case of PMMA. Observation (i) can be explained by the fact that the pyridyl group enables coordination to the $\mathrm{SiO}_{2}$ surface via the pyridyl lone pair orbital. This is supported by the observation that when using PBI without a functional pyridyl substituent (DAP), we observe a comparable number of PBI molecules per area as compared to PP in PMMA. In case that coordination to the $\mathrm{SiO}_{2}$ surface occurs via the pyridyl substituent, $\mathrm{PP}$ should with respect to the long molecular axis be orientated almost perpendicular to the surface. Since the long molecular axis coincides with the orientation of the optical $\mathrm{S}_{0}-\mathrm{S}_{1}$ transition dipole moment, the transition probability both in absorption and emission should be weak. This will result - in comparison with PMMA (assuming a random orientation of PP) in less intense single PP emitters. This is in fact observed experimentally and explains observation (ii).

As a next step we will discuss PP fluorescence energies in various environments. In PMMA we observe for the quasi-ensemble spectrum an apparent blue shift of the fluorescence as compared to the spectra for PP in toluene and on $\mathrm{SiO}_{2}$. Since PMMA and toluene are similar to each other with respect to polarity and dielectric properties, solvatochromic shifts can be safely excluded. This implies that the observed differences are related to differences in the distribution of the conformations of PP. Both, in toluene and on $\mathrm{SiO}_{2}$ the motion of PP molecules will be less restricted and an energetically broader range of conformations will be realized. However, the quasi-ensemble spectrum for $\mathrm{PP}$ on $\mathrm{SiO}_{2}$ clearly shows a similar range of conformations as compared to PMMA.

This qualitative finding is confirmed quantitatively upon inspection of Figure 4 which clearly demonstrates several distinct maxima in the fluorescence energy distribution, which we assign to different conformations. The distribution is broader and more red-shifted in case of $\mathrm{SiO}_{2}$ as compared to PMMA. In the following, the aforementioned findings of the recent publication by Fron et al. $[10,11]$ will be discussed in some detail to aim at a deeper understanding of our experimental results.

Hofkens et al. have previously assigned at least two distinctly different limiting cases of bay group controlled PBI conformations [2,9]. In a subsequent study Fron et al. [11] extended the model of bay group controlled conformations via supporting theoretical calculations considering precisely the tilt of each individual phenoxy (bay) group. $\mathrm{MD}_{\mathbf{z}}$ simulations of $\mathrm{PBI}$ molecules taking the solvent toluene into account have shown, that two limiting
Deleted: in an

Deleted: direction

Deleted: at 
conformations exist, namely a with respect to the bay group orientation fully folded and extended form. They could show, that the extended form is the most stable (ground state) conformation in toluene, while interconversion between folded and extended forms takes place, in close agreement with optical time resolved experiments, on (calculated time) scales of about 500 ps. However, the semiempirical Hartree-Fock calculations of the ground states of PBI type molecules in vacuum showed, that the folded conformation becomes stabilized. Excited state properties have been calculated without taking the solvent into account. Doing so, transition energies and radiative lifetimes have been obtained for each conformation [11]. Hence the most folded conformation should be lowest in fluorescence energy, while the less folded one is at the higher energy. Additionally ${ }_{2}$ the more folded conformations have been associated with increasing radiative lifetimes.

Single photon timing and transient absorption experiments revealed a spectral shift from $<590 \mathrm{~nm}$ to more red emitting conformations within a time range of $500 \mathrm{ps}$. Since MD simulations show more frequent transitions from folded to extended conformations and single photon time experiments indicate conversion from short to long wavelengths the extended conformation in the excited state was expected to be lowest in energy. However, calculated excited state energies showed the opposite behaviour. This contradiction has been removed by taking solvent shifts into account thus lowering the extended PBI conformation energetically below the folded one.

Next we compare our present experiments in relation to those recent findings. With respect to our results we identify the multimodal distribution of spectral positions of PP with discrete conformations controlled by the bay groups as suggested. Under these assumptions inspection of Figure 4 clearly shows, that on $\mathrm{SiO}_{2}$ more folded conformations are realized as compared to PP in PMMA. Since PP will be less hindered on $\mathrm{SiO}_{2}$ with respect to interconversion dynamics the situation resembles more closely the "vacuum-type" situation described by Fron et al. We therefore assume that the calculated fluorescence red shift holds for a folded conformation.

The situation might become different for PP in PMMA since Fron et al. predict, that upon solvent-solute interaction the extended form should be shifted energetically below the folded one. Thus they obtain both in ground and excited state a stabilisation of the bay groups in agreement with an extended conformation. However, we feel that this assumption might be abandoned while allowing for a different stabilisation energy for the bay groups in the ground and excited state. Why this suggestion holds, will be discussed later on in relation with our
Deleted: in toluene

Deleted: b

Deleted: on opposite 
findings for the wavelength dependent fluorescence intensities observed for single PP molecules.

The question immediately arises whether an interconversion between different conformations can be observed experimentally both on $\mathrm{SiO}_{2}$ and in PMMA. We will discuss this issue in relation to results presented in Figure 5. From comparison it is evident, that most of the spectral jumps occur both for $\mathrm{SiO}_{2}$ and PMMA in steps less than $\pm 5 \mathrm{~nm}$ which is certainly smaller than the wavelength difference between different conformations which are in the range of 10 to $15 \mathrm{~nm}$. However, in both cases we find spectral jumps larger than $10 \mathrm{~nm}$. Such large jumps occur about two times more often for $\mathrm{SiO}_{2}$ as compared to PMMA (see insets in Figure 5). This implies that interconversion between different conformations is possible in both environments though less frequent in PMMA. Spectral shifts related to a conformational change (>10nm) on $\mathrm{SiO}_{2}$ occur more often in form of blue-shifts. Obviously the direction of interconversion occurs in both directions.

Deleted: there is
Deleted: such
Deleted: point
Deleted: detected
Deleted: ranges

Deleted: ranges

Deleted:

Deleted: ranges with $\lambda_{\max }$

Deleted:

Deleted: works

In Figure 3 we have shown that besides the well known fluorescence intensity (on-off) blinking, variations in fluorescence intensity can be observed. We now like to discuss, what the possible origins of such variations are and whether intensity fluctuations are correlated to spectral jumps or spectral diffusion. The correlation between single molecule intensity and wavelength are shown in Figure 7. As can be seen, there is in fact a correlation in case of PMMA and $\mathrm{SiO}_{2}$. Both, for PMMA and $\mathrm{SiO}_{2}$ the intensity decreases below about $590 \mathrm{~nm}$ with decreasing wavelength while it also decreases above $594 \mathrm{~nm}$ resulting in a pronounced maximum at about $590-595 \mathrm{~nm}$.

Figure 7 Correlation of fluorescence intensities and fluorescence peak wavelengths of all PP spectra observed on $\mathrm{SiO}_{2}$ and in PMMA, respectively.

How can such a variation of fluorescence intensities be explained? According to calculations of Fron et al. the radiative lifetime of $\mathrm{PB}$ d depends critically on the type of conformation. The general observation is that the folded conformation is related to a longer radiative lifetime, while shorter lifetimes correspond to the more extended ones. When neglecting non-radiative Deleted: s

Deleted: type molecules decay processes for a moment short radiative lifetimes correspond to high fluorescence intensities. This assumption will explain our finding that above $590 \mathrm{~nm}$ the PP fluorescence intensities are decreasing with wavelength, since we approach the most folded conformation at the lowest fluorescence energy. Unfortunately, similar arguments cannot explain the 
decrease of fluorescence intensities below $590 \mathrm{~nm}$. According to Fron et al. $[10,11]$ the extended conformation (at high energies according to calculations in vacuum) should be related to the short radiative lifetime and thus to the highest fluorescence intensity. However, keeping in mind that interconversion takes place from extended conformations (at high energies) to folded ones (at lower energies), this introduces a non-radiative decay channel as has been experimentally observed by Fron et al. Therefore we suggest, that the fluorescence intensity decrease below $590 \mathrm{~nm}$ is related to such a non-radiative interconversion channel. As compared to experiments in solution the time scale will be certainly slowed down in PMMA and might to some extent also be hindered on $\mathrm{SiO}_{2}$ due to surface attachment. This way radiative decay will compete with the interconversion rate. Contrary to arguments posed by Fron et al. recently, we state - following our interpretation _of fluorescence intensities that the extended form is as calculated by INDO methods energetically higher than the folded one. Those calculations have shown [11], that in vacuum the folded conformation becomes stabilized. These results would be clearly supported by our results for $\mathrm{PP}$ on $\mathrm{SiO}_{2}$, for which solvent solute interactions will be less important. To explain our findings in PMMA only needs the assumption that stabilisation energies in ground and excited state conformations become different, stabilizing an extended form in the ground state, while in the excited state the folded one is lower in energy in accordance with vacuum calculations. Such changes might be driven by changing the overall "planarity" of the PBI chromophore upon optical excitation.

Summarizing this part related to the wavelength - intensity correlation shown in Figure 7, molecules emitting at wavelengths longer than $592 \mathrm{~nm}$ are supposed to be in a more folded conformation resulting in decreased intensities due to the increase of the radiative lifetime as expected from INDO calculations. Molecules emitting at wavelengths shorter than $592 \mathrm{~nm}$ exhibit a non-radiative decay, most likely being due to interconversion dynamics.

In conclusion, PBI molecules show strong variations in fluorescence intensities which might be assigned to both conformation changes (accompanied by spectral jumps larger that $10 \mathrm{~nm}$ ) and/or fluctuations of the environment which result in jumps more closely to $5 \mathrm{~nm}$, Since we observe conformational changes also for "free" $\mathrm{PP}$ on $\mathrm{SiO}_{2}$, such transitions are obviously not only driven by fluctuations of the dye-solvent interaction, but might be basically due to "intra molecular" conformational transitions. In such a situation the intensity-to-wavelength

Deleted: glassy
Deleted: still

Deleted: in

Deleted: stabilisation energies 
relationship will become complex, since different conformations have different quantum yields.

Intensity fluctuations may also be caused by variations in the absorption cross section (change of the spectral position of the absorption band, orientation of the transition dipole of the absorption band or change of the absorption band-width). The latter can be ruled out since $\underline{\text { band-widths of all recorded spectra show no significant change, whereas absorption band }}$ shifts (of about 10nm) may influence intensity at most by a factor 2 at a constant excitation wavelength of $465 \mathrm{~nm}$. The role of molecular dipole orientation has already been discussed earlier in this paper. Hence changes in the molecular orientation are expected to be distributed rather arbitrary. In summary, variations in the absorption cross section are not the major contributions to the overall changes mentioned in the discussion related to the results presented in Figure 7.

While PP conformation changes will be strongly hindered in PMMA they are expected to be less hindered for PP on $\mathrm{SiO}_{2}$. Therefore we will now discuss possible motional schemes as identified in combination with polarisation experiments with special regard to the role of the functional pyridyl group. From polarisation dependent fluorescence detection (see Figure 6), we obtain in case of single PP emitters additional information upon the orientation. It is immediate obvious, that PP molecules show in PMMA on time scales of seconds only small changes in polarisation and thus orientation.

The situation is completely different for $\mathrm{PP}$ on $\mathrm{SiO}_{2}$. Besides the preferential orientation of PP on $\mathrm{SiO}_{2}$ large jumps in polarisation are observed for $\mathrm{SiO}_{2}$, whereas variations in PMMA occur very smoothly. However, especially on $\mathrm{SiO}_{2}$ the jumps between the two polarisation channels are not in all cases anti-correlated, as has in contrary been reported for several cases of rotational motion of single molecules $[5,15]$. This supports the model that we have a kind of restricted motion caused by the coordination of PP via the pyridyl functional group to the $\mathrm{SiO}_{2}$ surface.

As already mentioned, experiments on single PP molecules on $\mathrm{SiO}_{2}$ indicate that pyridyl substitution enhances surface attachment which has to be characterized by a nearly perpendicular orientation of the long molecular axis to the $\mathrm{SiO}_{2}$ surface. Since the long $\mathrm{z}$-axis carries the main optical dipole transition moment, excitation and emission will be reduced considerably in accordance with experimental observations. Figure 8 shows under the assumption of a nearly perpendicular orientation and neglecting any $\mathrm{x}$ - or $\mathrm{y}$-polarisation the 
allowed schemes of motion with respect to polarisation. Only two motions will be of importance. (i) Tilting from a nearly perpendicular orientation (of the z-axis) into the $\mathrm{SiO}_{2}$ plane will result in a synchronous increase of both s- and p-polarisation. The relative s- and pintensities will depend on the respective projections of the in-plane z-component onto these axes, which are characterized by $\phi$. (ii) Rotation of the effective in-plane component around the normal to the $\mathrm{SiO}_{2}$ plane will result in an alternating s- and p-polarisation. Combinations of type (i) and (ii) motions will result in a more complex time dependent fluorescence polarisation.

Figure 8 Scheme for the orientation and related motional schemes of $\mathrm{PP}$ on the $\mathrm{SiO}_{2}$ surface (left). Polarisation intensities of molecule (b) (see Figure 6) in the time range between 60 and $80 \mathrm{~s}$ (right).

Figure 6 shows various examples of time evolution scenarios of the polarisation and confirms our model of a nearly perpendicular orientation of $\mathrm{PP}$ on $\mathrm{SiO}_{2}$. The typical behaviour of $\mathrm{PP}$ on $\mathrm{SiO}_{2}$ and in PMMA films is described by the six time traces ((a)-(c) and (d)-(f), respectively). A major fraction of single PP molecules exhibits almost no fluctuation in polarisation both on $\mathrm{SiO}_{2}$ (a) and in PMMA films (d). A second smaller fraction of molecules suddenly jumps in polarisation in case of $\mathrm{SiO}_{2}((\mathrm{~b}),(\mathrm{c}))$, whereas in PMMA changes in polarisation are much lower in magnitude and gradual in time ((e),(f)).

In case of $\mathrm{SiO}_{2}$, PP molecules (b) and (c) in Figure 6 show a tilt (synchronous increase of pand s-polarisation). Molecule (b) shows additionally strong fluctuations of the polarisation (alternating p- and s-polarisation) in the time range between 60 and $80 \mathrm{~s}$. This alternating polarisation is shown on an enlarged scale in Figure 8, which clearly proofs an in-plane rotation accompanied by a tilt of the polarisation axis. All examples we investigated with respect to polarisation changes show similar dynamics which can be explained in terms of both tilt and in plane-rotation of the long molecular axis. For $\mathrm{PP}$ on $\mathrm{SiO}_{2}$ jumps in polarisation take place instantaneously on the experimental time scale of $100 \mathrm{~ms}$. Distributions for $\phi$ shown for molecules (a) and (d) are related to statistically fluctuating polarisation intensities. Only PP on $\mathrm{SiO}_{2}$ (molecules (b) and (c)) show a considerably larger $\phi$ distribution.

The polarisation behaviour is completely different for PP in PMMA, where changes in polarisation are hardly to observe (see Figure 6) and occur only gradually with time. This proves that rotational dynamics of PP are nearly frozen in PMMA, but nevertheless PP might 
undergo conformational dynamics (identified by spectral jumps) within the polymer cage. It should be mentioned, that changes in polarisation might be also caused by interconversion between conformations in case, that they are accompanied by bending of the effective PP chromophoric unit. This has been demonstrated by calculations of Fron et al. in case of the folded conformation. Further experiments are needed on a single molecule level in order to discriminate between these two processes. 
Different molecular conformations related to the bay group orientation have been reported previously for various PBI-type molecules [9-11,26]. Also in the present experiments we were able to detect various conformations and related interconversions which are accompanied by changes in the fluorescence quantum yield. In PMMA the distribution of conformations is shifted towards the more extended forms in the short wavelength region. Our findings can, with some modifications be interpreted in terms of recently published experiments and MD simulations [11]. Additionally, our single molecule detection scheme allows for a mechanistic interpretation of such complex dynamics.

We could identify two ranges of spectral shifts, namely large ones which are probably related to interconversion between different conformations and small wavelength jumps. This indicates, that small spectral jumps are not in all cases controlled only by the dynamics of the environment, since $\mathrm{SiO}_{2}$ surfaces and PMMA polymers are subject to completely different dynamics of the environment. An open question still is, whether conformation changes are systematically related to rotational motions. We have not yet been able to correlate e. g. intensity fluctuations with changes in orientation. This is a crucial information since intensity fluctuations might be caused by changes in conformation or/and orientation and additional (e. g. photochemical) blinking processes.

Further experiments are planned to correlate systematically fluorescence lifetimes, orientation (polarisation) and spectral shifts to provide a strictly mechanistic model of molecular motion 
on various surfaces and $\underline{i n}$ matrices. Such information can be obtained uniquely with a single

Deleted: on molecule level detection scheme. 


\begin{abstract}
Acknowledgments
We like to thank Frank Würthner and Rainer Dobrawa (University of Würzburg) for a kind donation of compounds PP and DAP and acknowledge financial support by the Volkswagen Foundation within the program "Single Molecules in Physics, Chemistry and Biology" (Contract No. I/79435).
\end{abstract}


Table of figures

Figure 1 Molecular structures of pyridyl- and alkyl-functionalized PBI derivatives PP (left) and DAP (right). One of the four phenoxy groups in the PBI's so-called bay area is exemplarily marked at the PP molecule. .4 Figure 2 Fluorescence spectra of PP in different environments. Black (PMMA) and red $\left(\mathrm{SiO}_{2}\right)$ spectra result from summing up the respective single molecule spectra recorded during a 1 min measurement period. Excitation wavelength was $532 \mathrm{~nm}$..... ... 6

Figure 3 Temporal evolution of PP fluorescence on $\mathrm{SiO}_{2}$ (left) and in PMMA (right). The integrated fluorescence intensity (determined by a fit to two Gaussian lines at the fluorescence origin and the vibronic side band, respectively) are shown as traces on the right of each spectral scan. The centres of the two fitted spectral positions (traces on the left) of the respective spectra are also indicated. After about $55 \mathrm{~s}$ (left) and $65 \mathrm{~s}$ (right) PP molecules are | photobleached permanently 6.

Figure 4 Distribution of fluorescence maxima $\lambda_{1}$ position for single PP molecules on $\mathrm{SiO}_{2}$ and in thin PMMA films. The insets show the spectral position of $\lambda_{2}$ vibrational bands relative to $\lambda_{1}$. Broken lines indicate possible PP conformations below and above $592 \mathrm{~nm}$, which

| corresponds to the wavelength with the highest fluorescence intensity (see Figure 7). 7

Deleted: 8 Figure 5 Distribution (on two probability scales) of spectral jumps occurring between two subsequently recorded spectra (1s binning time for each spectrum). The insets show the largest spectral range $\Delta \lambda_{\max }$ covered by one individual molecule during the total observation | time. $\underline{7}_{-}$

Deleted: 9

Figure 6 Representative time resolved polarisation intensities for 3 single molecules of PP on $\mathrm{SiO}_{2}$ surfaces ((a) - (c)) and in a PMMA thin film ((d) - (f)), respectively. Channel 1 and 2 correspond to fluorescence intensities of the two perpendicular in-plane polarisation directions (top trace). $\phi$ (middle trace) corresponds to the time dependence of the projection angle $\phi$ (see Figure 8). The lowest trace shows the probability distribution of the related $\phi$ | time trace above). Figure 7 Correlation of fluorescence intensities and fluorescence peak wavelengths of all PP spectra observed on $\mathrm{SiO}_{2}$ and in PMMA, respectively.

\section{P.} Figure 8 Scheme for the orientation and related motional schemes of $\mathrm{PP}$ on the $\mathrm{SiO}_{2}$ surface (left). Polarisation intensities of molecule (b) (see Figure 6) in the time range between 60 and | 80 s (right). 
${ }^{1}$ H. Yang, G. Luo, P. Karnchanaphanurach, T.-M. Louie, I. Rech, S. Cova, L. Xun, X. Sunney Xie, Science 302, 262 (2003).

${ }^{2}$ Renaud A. L. Vallée, Mircea Cotlet, Mark Van Der Auweraer, Johan Hofkens, K. Müllen, Frans C. De

Schryver, JACS 126, 2296 (2004).

${ }^{3}$ Michael W. Holman, Ping Yan,Koon-Cheung Ching, Ruchuan Liu, Fady I. Ishak and David M. Adams, Chem. Phys. Lett. 413, 501 (2005).

${ }^{4}$ L.A. Deschenes, D.A. Vanden Bout, Science 292, 255 (2001).

5 A. Schob, F. Cichos, J. Schuster, C. von Borczyskowski, Eur. Polymer J. 40, 1019 (2004).

${ }^{6}$ Eigen, M., and Rigler, R. PNAS USA 91, 5740 (1994).

${ }^{7}$ J. Schuster, F. Cichos, C. von Borczyskowski, Eur. Polymer J. 40, 993 (2004).

${ }^{8}$ J. Schuster, F. Cichos, C. von Borczyskowski, EPJ E - Soft Matter 12, 75 (2003).

${ }^{9}$ J. Hofkens, T. Vosch, M. Maus, F. Köhn, M. Cotlet, T. Weil, A. Herrmann, K. Müllen, F.C. De Schryver, Chem. Phys. Lett. 333, 255 (2001).

${ }^{10}$ E. Fron, R. Pilot, G. Schweitzer, J. Qu, A. Herrmann, K. Müllen, J. Hofkens, M. Van der Auweraer and F. C. De Schryver, Photochem. Photobiol. Sci.. 7, 597 (2008).

${ }^{11}$ E. Fron, G. Schweitzer, P. Osswald, F. Würthner, P. Marsal, D. Beljonne, K. Müllen, F. C. De Schryver , M.

| Van der Auweraer, Photochem. Photobiol. Sci. 7 1509 (2008),

${ }^{12}$ H. Langhals, Heterocycles 40, $4 \overline{7} \overline{(1995)}$.

${ }^{13}$ P. Osswald, D. Leusser, D. Stalke, F. Würthner, Angew. Chem. Int. Ed. 44, 250 (2005).

$\mid{ }^{14}$ M. Cotlet, S. Masuo, G. Luo, J. Hofkens, M. Van der Auweraer, J. Verhoeven, K. Müllen, X. Sunney Xie, F. De Schryver, PNAS 101, 14343 (2004).

${ }^{15}$ N. Tomczak, R. A. L. Vallée, E. M. H. P. van Dijk, M. García-Parajó, L. Kuipers, N. F. van Hulst, G. Julius Vancso, Eur. Polymer J. 40, 1001 (2004).

${ }^{16}$ R.A.L. Vallée, M. Cotlet, J. Hofkens, and F. C. De Schryver, Macromolecules 36, 7752 (2003).

${ }^{17}$ R.A.L. Vallée, W Paul, K Binder, J. Chem. Phys. 127, 154903 (2007).

${ }^{18}$ F. Würthner, A. Sautter, D. Schmid, P. J.A.Weber, Chem. Eur. J. 7, 894 (2001).

${ }^{19}$ E. Lang, F. Würthner, J. Köhler, ChemPhysChem 6, 935 (2005).

${ }^{20}$ D. Kowerko, J. Schuster, N. Amecke, M. Abdel-Motaleb, R. Dobrawa, F. Würthner, C. von Borczyskowski, ChemPhysChem, subm.

${ }^{21}$ R. Dobrawa, F. Würthner, Chem. Comm. 2002, (1878).

${ }^{22}$ E. Lang, R. Hildner, H. Engelke, P. Osswald, F. Würthner, J. Köhler, ChemPhysChem 8, 1487 (2007).

${ }^{23}$ X. Michalet, S. Weiss, C. R. Physique 3, 619 (2002).

${ }^{24}$ F. Würthner, C. Thalacker, S. Diele, C. Tschierske Chem. Eur. J. 7, 2245 (2001).

${ }^{25}$ F. Würthner, Pure Appl. Chem. 78, 2341 (2006).

${ }^{26}$ P. Osswald and F. Würthner, Chem. Eur. J. 13, 7395 (2007).

${ }^{27}$ M. Lippitz, C.G. Huebner, T. Christ, H. Eichner, P. Bordat, A. Herrmann, K. Müllen, T. Basche, Phys. Rev. Lett. 92, 103001 (2004).

${ }^{28}$ R. Dobrawa, M. Lysetska, P. Ballester, M . Grüne, F. Würthner, Macromolecules 38, 1315 (2005).

Formatted: Font: Not Italic Formatted: Font: Bold, Not Italic 

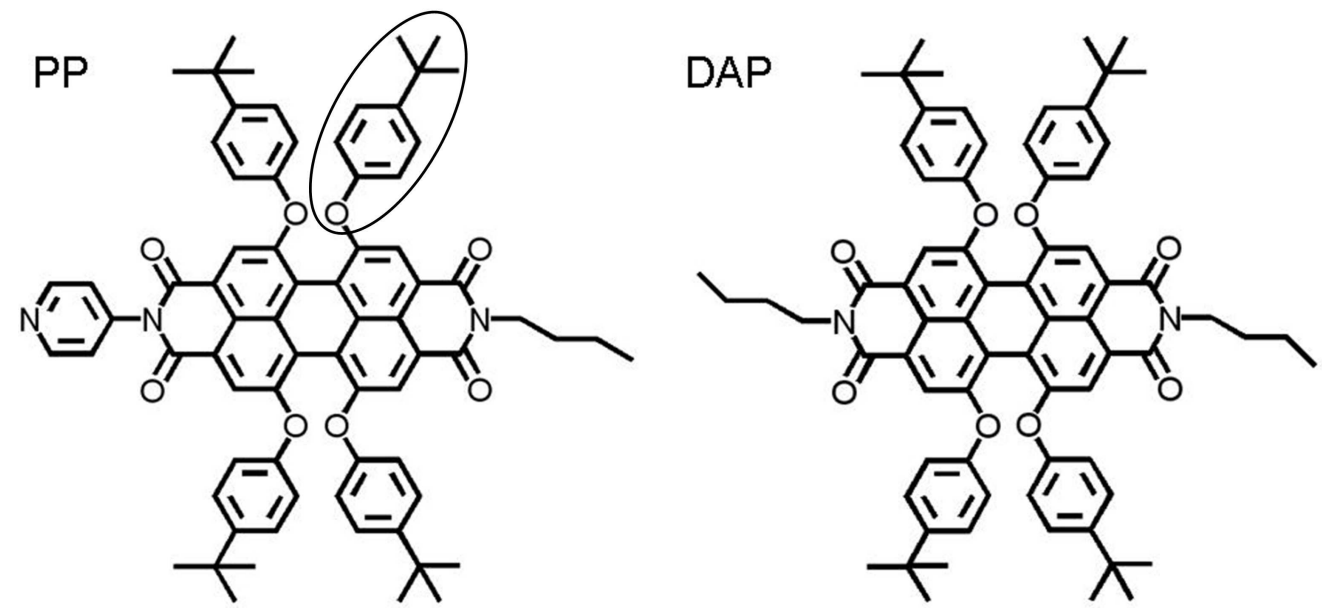

Molecular structures of pyridyl- and alkyl-functionalized PBI derivatives PP (left) and DAP (right). One of the four phenoxy groups in the PBI's so-called bay area is exemplarily marked at the PP molecule.

$254 \times 117 \mathrm{~mm}(307 \times 307 \mathrm{DPI})$ 


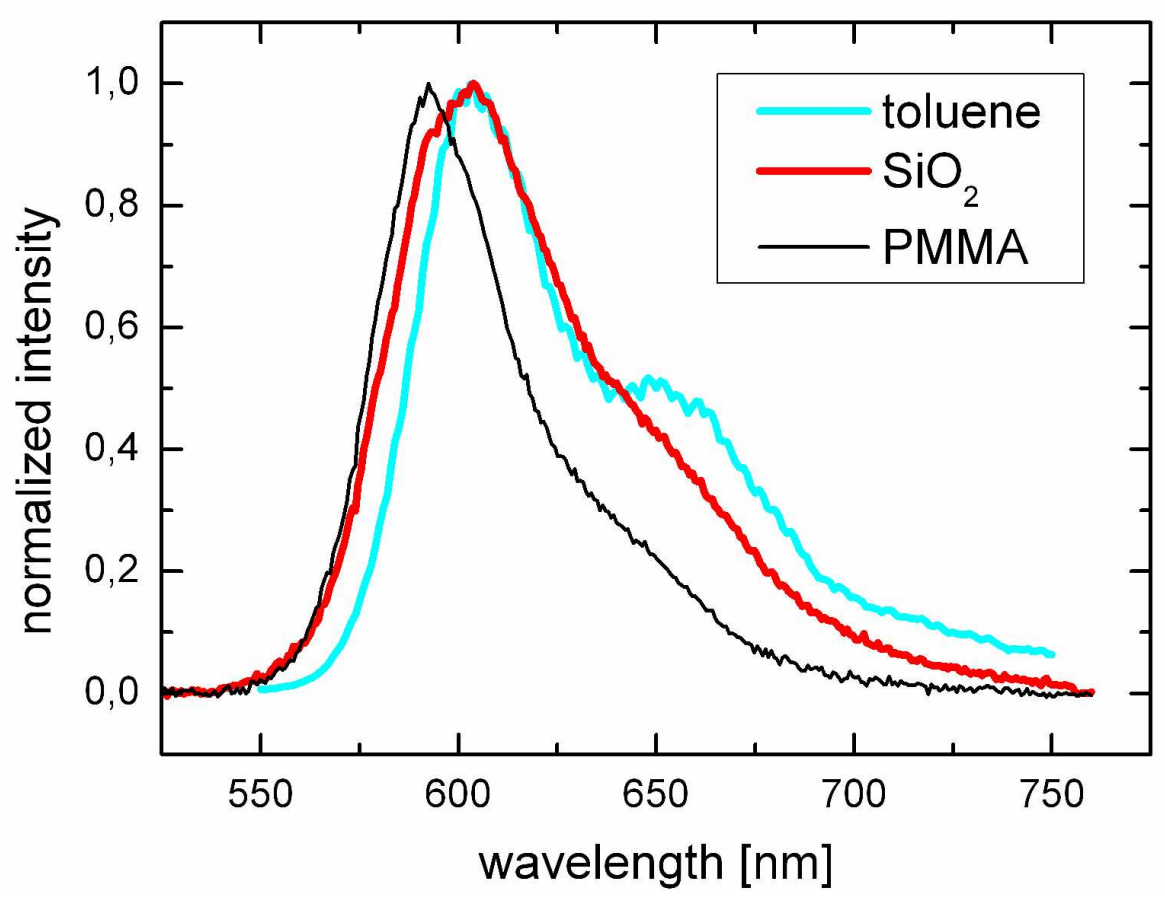

figure 2 Fluorescence spectra of PP in different environments. Black (PMMA) and red $\left(\mathrm{SiO}_{2}\right)$ spectra result from averaging of the respective single molecule spectra recorded during a $1 \mathrm{~min}$ measurement period. Excitation wavelength was $532 \mathrm{~nm}$. $289 \times 203 \mathrm{~mm}(300 \times 300 \mathrm{DPI})$ 


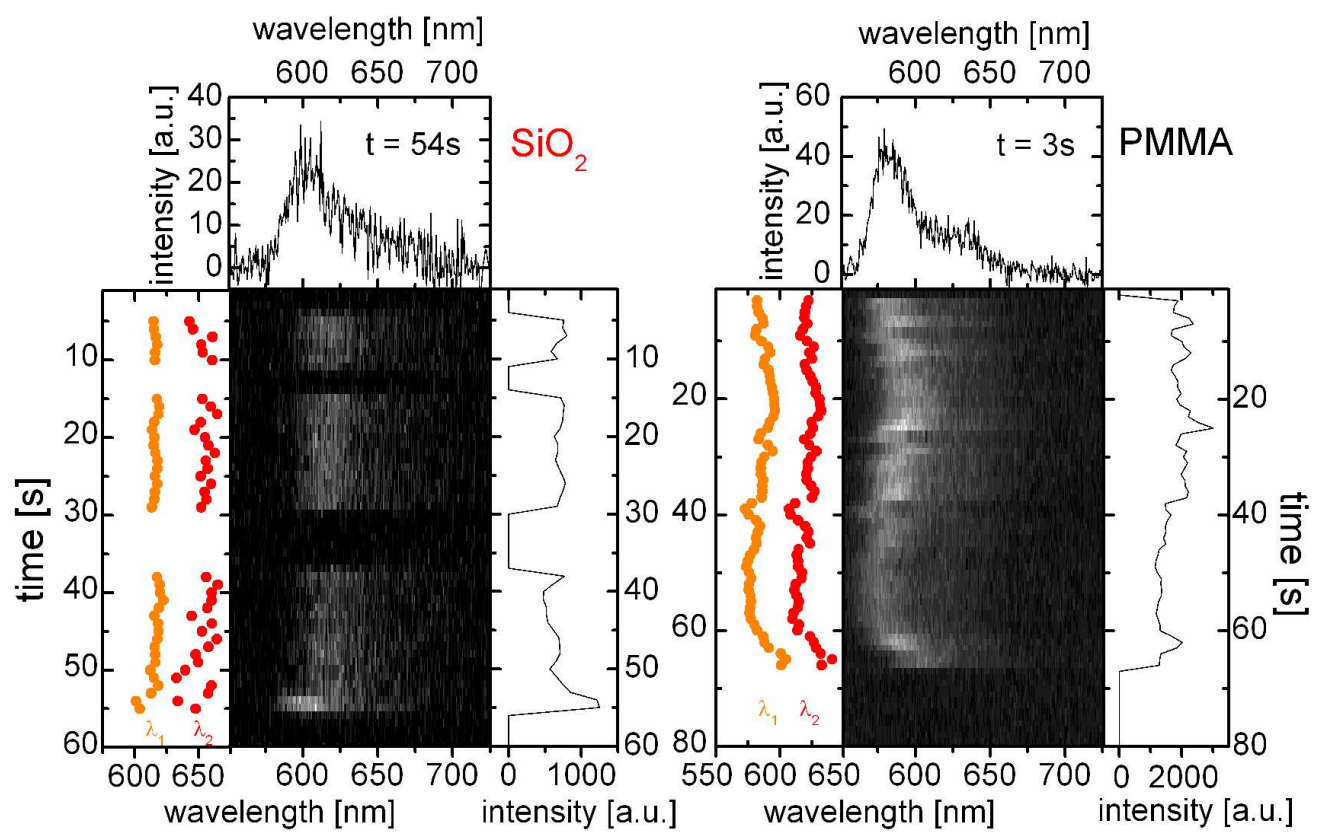

figure 3 Temporal evolution of PP fluorescence on $\mathrm{SiO}_{2}$ (left) and in PMMA (right). The integrated fluorescence intensity (determined by a fit to two Gaussian lines at the fluorescence origin and the vibronic side band, respectively) are shown as traces on the right of each spectral scan. The centres of the two fitted spectral positions (traces on the left) of the respective spectra are also indicated. After about $55 \mathrm{~s}$ (left) and $65 \mathrm{~s}$ (right) PP molecules are photobleached. $289 \times 203 \mathrm{~mm}(300 \times 300 \mathrm{DPI})$ 


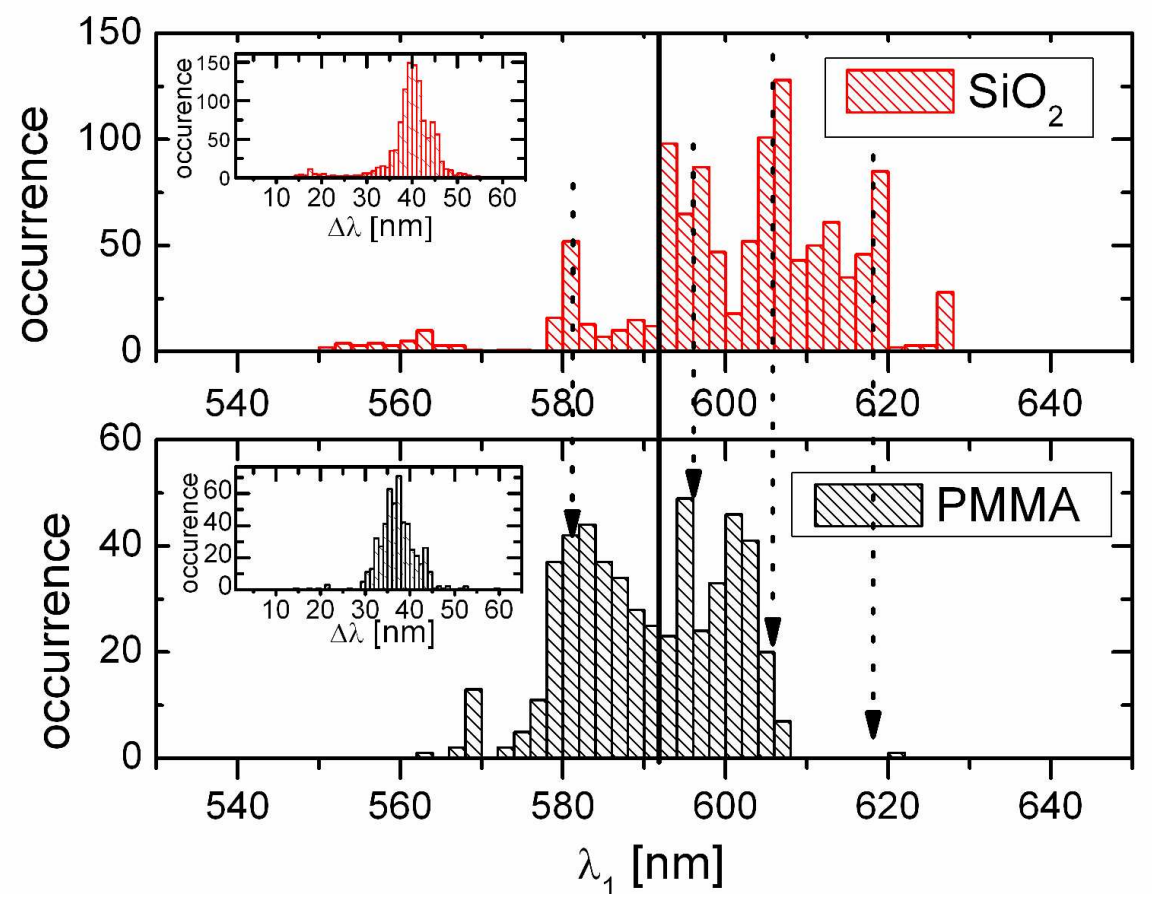

figure 4 Distribution of $\lambda_{1}$ position for single PP molecules on $\mathrm{SiO}_{2}$ and in thin PMMA films. The insets show the spectral position of $\lambda_{2}$ relative to $\lambda_{1}$. Broken lines indicate possible PP conformations below and above $592 \mathrm{~nm}$, which corresponds to the wavelength with the highest fluorescence intensity (see Figure 7). $289 \times 202 \mathrm{~mm}(300 \times 300$ DPI $)$ 

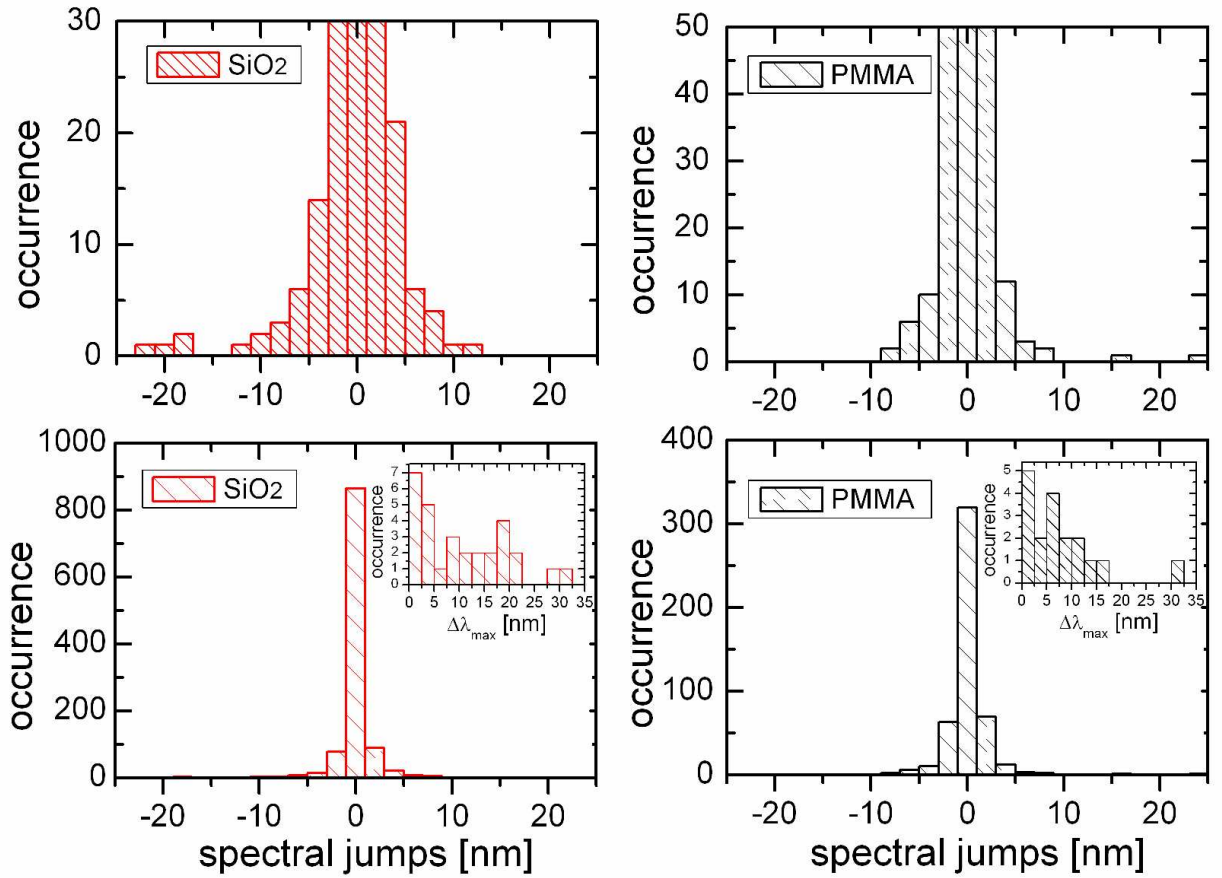

figure 5 Distribution of spectral jumps (on two probability scales) occurring between two subsequently recorded spectra (1s binning time for each spectrum). The insets show the largest spectral range $\Delta \lambda_{\max }$ observed for one individual molecule during the total observation time $289 \times 202 \mathrm{~mm}(300 \times 300$ DPI $)$ 

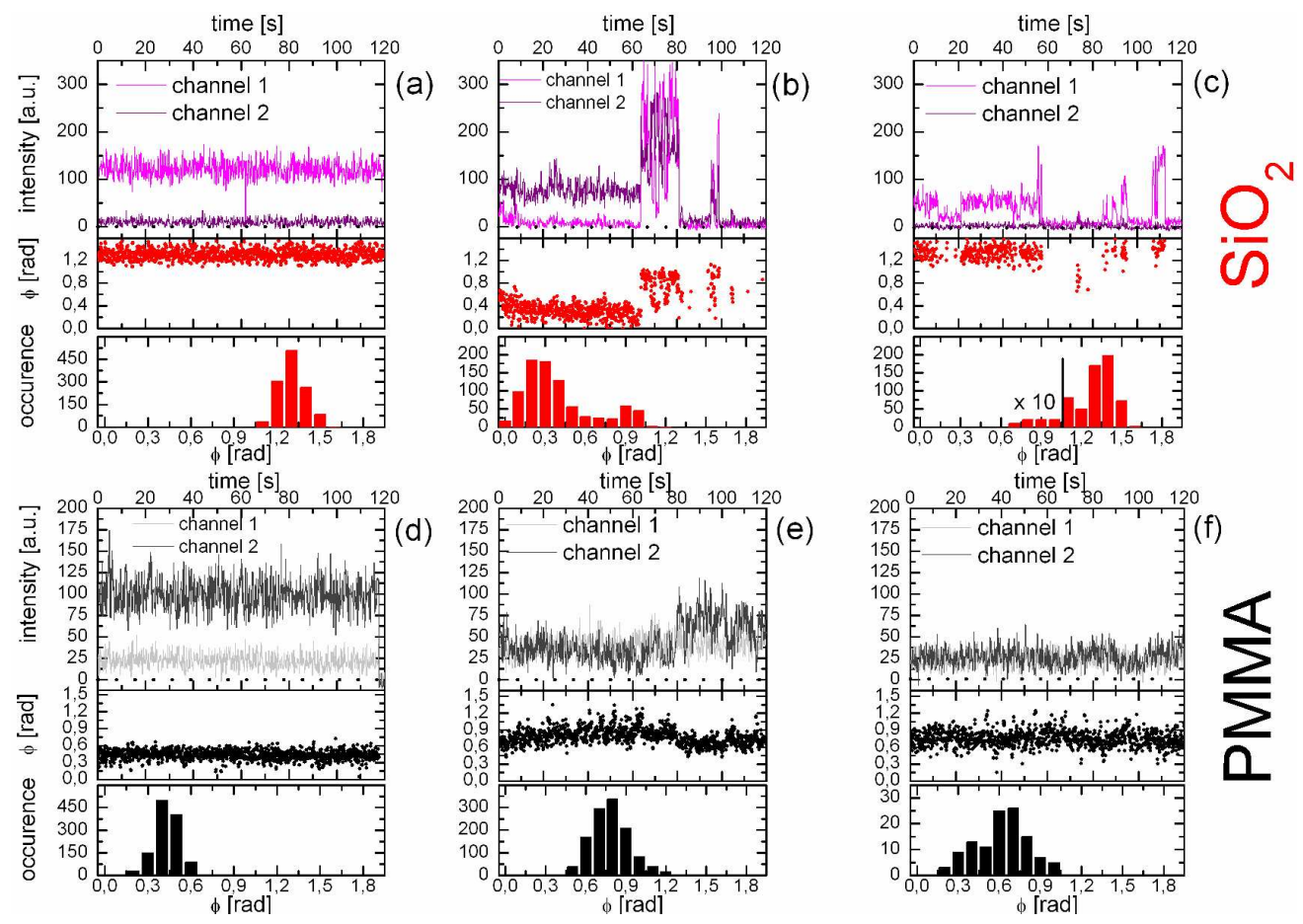

figure 6 Typical polarisation intensities resolved in time for 3 single molecules of $\mathrm{PP}$ on $\mathrm{SiO}_{2}$ surfaces $((a)$ - (c)) and in a PMMA thin film ((d) - (f)), respectively. Channel 1 and 2 corresponds to fluorescence intensities of the two perpendicular in-plane polarisation directions (top trace). $\phi$ (middle trace) corresponds to the time dependence of the projection angle $\phi$ (see Figure 8 ). The lowest graph shows the probability distribution of the related $\$$-time trace (middle trace) $289 \times 203 \mathrm{~mm}(300 \times 300$ DPI $)$ 


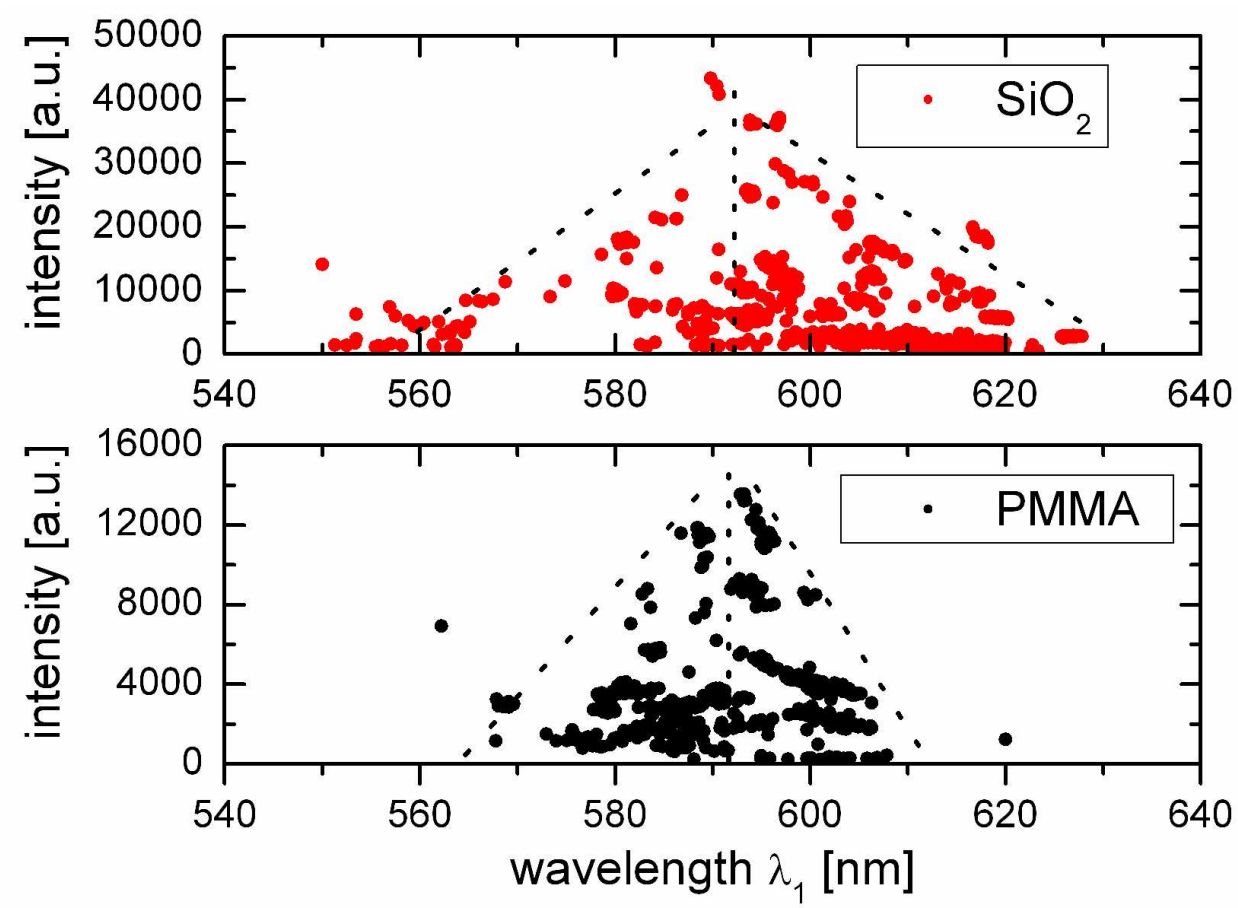

figure 7 Correlation of fluorescence intensities and fluorescence peak wavelengths of all PP spectra observed on $\mathrm{SiO}_{2}$ and in PMMA, respectively $289 \times 202 \mathrm{~mm}(300 \times 300 \mathrm{DPI})$ 


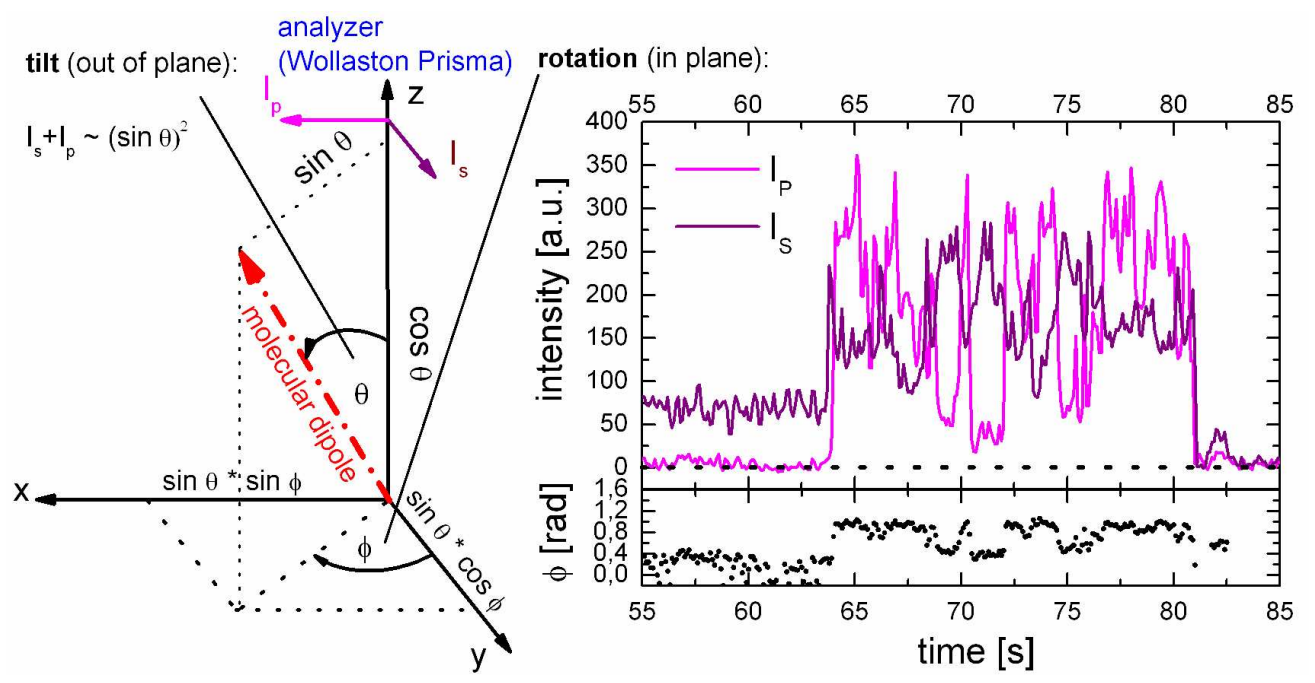

figure 8 Scheme for the orientation and related motional schemes of PP on the $\mathrm{SiO}_{2}$ surface (left). Polarisation intensities of molecule (b) (see Figure 6) in the time range between 60 and $80 \mathrm{~s}$ (right) $289 \times 147 \mathrm{~mm}(300 \times 300$ DPI) 